\title{
A Simple Thermal Conductivity Model for Unsaturated Geomaterials Accounting for Degree of Saturation and Dry Density
}

\author{
Agostino Walter Bruno $^{1}$ (D) . Doaa Alamoudi ${ }^{1}$
}

Received: 14 August 2020 / Accepted: 27 September 2020 / Published online: 16 October 2020

(c) The Author(s) 2020

\begin{abstract}
This paper proposes a simple thermal conductivity model for geomaterials accounting for the combined effect of both degrees of saturation and dry density. The model only requires the determination of the thermal conductivity under dry conditions (i.e., at a degree of saturation equal to zero) and as little as two additional measurements of thermal conductivity performed at different levels of degree of saturation and dry density. The model is a function of only two fitting parameters, namely the moisture factor $m_{f}$ and the density factor $m_{d}$. Despite its simplicity, the model can correctly predict the thermal conductivity of geomaterials and this has been validated against five sets of experimental data obtained on a very broad range of materials ranging from fine (e.g., bentonite) to coarser soils (e.g., a mix of gravel, coarse sand and silt) tested at different levels of degree of saturation and dry density. The paper also shows that the model can be applied to different engineering contexts such as (a) the thermal behaviour of earth materials used for building construction, (b) the thermal performance of bentonites employed for the storage of nuclear waste and (c) the estimation of the heat exchange in shallow geothermal reservoirs. Finally, the proposed model can be easily implemented in a finite element code to perform numerical simulations to study the heat transfer in unsaturated geomaterials.
\end{abstract}

Keywords Thermal conductivity · Geomaterials · Partial saturation · Dry density $\cdot$ Heat transfer

\section{Introduction}

The thermal conductivity of unsaturated geomaterials plays a fundamental role in a broad range of engineering, geophysical and geoenvironmental applications such as (a) the storage and recovery of heat from shallow geothermal reservoirs [1-4]; (b) the thermal performance of bentonitic clay used for the storage of highly radioactive nuclear waste [5-7]; (c) the indoor thermal comfort inside earth dwellings [8, 9]; (d) management of crops and cultivations in agricultural applications [10,11].

Over the past few decades, the experimental measurement of the thermal conductivity of unsaturated porous geomaterials has enjoyed a significant progress and it can be obtained by means of various techniques involving either steady-state or transient methods [12]. The former group of

Agostino Walter Bruno

agostino.bruno@newcastle.ac.uk

1 School of Engineering, Geotechnics and Structures, Newcastle University, Devonshire Terrace, Drummond Building, Room 2.19, Newcastle upon Tyne NE1 7RU, UK methods encompasses various laboratory techniques, such as the absolute technique for which a sample is heated by a heat source with a known steady-state power and the resulting temperature gradient across the thickness of the material is monitored. Other steady-state methods also involve the use of comparative techniques (i.e., comparisons with materials of known thermal conductivity), measurements by means of heat flux meters, radial heat flow method and the parallel thermal conductance technique [12]. Instead, the transient methods, which encompass several laboratory techniques (e.g., pulsed power technique, hot wire method, transient plane source and laser flash thermal diffusivity method), overcome the issue of long waiting time for establishing steady-state conditions. The heat sources in transient methods function either periodically or as a pulse [12-14].

In parallel with the experimental advancement of the measuring techniques, various types of analytical models have been devised to predict the thermal conductivity of unsaturated geomaterials $[15,16]$. In particular, Dong et al. [15] subdivided the existing thermal conductivity models in the following three categories: (a) mathematical models, that are adopted from predictive models of other physical 
properties (such as dielectric permittivity, magnetic permeability, electric or hydraulic conductivity) which are calculated by considering the thermal conductivity of each component and its corresponding volume fraction; (b) mixing models, that conceptualise the multiphasic nature of geomaterials as a combination of solid, air and water blocks arranged either in series or in parallel, thus leading to lower and upper bounds of the thermal conductivity; (c) empirical models, that define a relationship between the relative thermal conductivity (i.e., normalised in relation to the saturated and dry thermal conductivities) and the water content (or equivalently the degree of saturation).

This paper firstly summarises some examples of these alternative approaches in modelling the thermal conductivity of soils available in the literature. Afterwards, a new and simple model to estimate the thermal conductivity of unsaturated geomaterials is proposed. Interestingly, this model accounts for the combined effect of the degree of saturation and dry density on the thermal conductivity of various geomaterials. The quality of the model predictions is successfully validated against five sets of experimental data, thus confirming its good capability of reproducing the thermal conductivity of different unsaturated geomaterials employed in different engineering applications (e.g., earth buildings, storage of nuclear waste, shallow geothermal reservoirs). Finally, the simplicity of the proposed model is such that it can be easily implemented into a numerical code (e.g., Finite Element Method software) to study the heat transfer in unsaturated geomaterials.

\section{Review of Existing Thermal Conductivity Models}

The moisture-dependent thermal conductivity $\lambda$ of a material is a measure of its ability to transfer or conduct heat and it is fundamental in numerous geotechnical, geophysical and geoenvironmental applications. This section revises some of the most used thermal conductivity models by considering the model classification proposed by Dong et al. [15].

A common approach in modelling the thermal conductivity of porous geomaterials in a partially saturated state is based on the consideration that the ability to transfer the heat of a given unsaturated geomaterial is dependent on the thermal conductivities and the volume fractions of solid grains, water and air. Based on this approach and as an example of mathematical models, De Vries [17] defined the following expression of the thermal conductivity $\lambda$ :

$\lambda=\frac{x_{a} \lambda_{a}+x_{w} \lambda_{w}+F x_{s} \lambda_{s}}{x_{a}+x_{w}+F x_{s}}$

where $\left\{x_{a} ; x_{w} ; x_{s}\right\}$ and $\left\{\lambda_{a} ; \lambda_{w} ; \lambda_{s}\right\}$ are respectively the volume fractions and the thermal conductivities of air, water and solid grains. The fitting parameter $F$ is a weighting factor which accounts for the continuity and shape of the solid particles and their effect on the thermal conductivity of the geomaterial. Given its dependency on both contact and arrangement of solid particles, it is interesting to note that the model parameter $F$ can also be seen as an implicit consideration of the effect of dry density on thermal conductivity. This model has the advantage of considering the effect of the degree of saturation for the two-pore fluids (i.e., air and water) as well as the volume fraction of the solid phase. On the other hand, the application of the model requires the knowledge of the individual thermal conductivities of the air, water and solid fractions. While the thermal conductivities of both air and water are well known, it can be challenging to determine the thermal conductivity of the solid fraction especially if the geomaterial is porous and composed by a mix of several mineralogical components.

In parallel, several mixing models are available in the literature and the most used are the ones proposed by Mickley [18], Woodside and Messmer [19] and McGaw [20]. In particular, Mickley [18] accounted for the heat conduction through a series of water films, solid and air blocks. Woodside and Messmer [19] considered instead of a three-component system of solid, water and air that is traversed by three parallel heat flow paths. This model required the thermal conductivity and volume fraction of each component and it only provided a good estimate of the thermal conductivity for saturated two-phasic materials [15]. Finally, McGaw [20] simplified the three-component system of Woodside and Messmer [19] by assuming the heat transfer does not occur at the particle contacts but only within the fluids. This simplifying assumption led to the introduction of an interfacial efficiency factor to be applied at the solid/fluid contact. The use of this model, therefore, requires the determination of the efficiency factor, which can generate uncertainty especially at the low values of degree of saturation [15].

Several empirical models were also proposed in the literature. Johansen [21] proposed the following expression for the thermal conductivity $\lambda$ of unsaturated soils:

$\lambda=\left(\lambda_{\mathrm{sat}}-\lambda_{\mathrm{dry}}\right) \lambda_{e}+\lambda_{\mathrm{dry}}$

where $\lambda_{\text {sat }}$ and $\lambda_{\text {dry }}$ represent the thermal conductivity of the soil under saturated and dry conditions, respectively. The term $\lambda_{e}$ represents instead the Kersten number. The saturated and dry thermal conductivities as well as the Kersten number for both fine- and coarse-grained soils are expressed by the following equations:

$\lambda_{\text {sat }}=\lambda_{s}^{(1-n)} \lambda_{w}^{n}$

$\lambda_{\text {dry }}=\frac{0.137 \rho_{d}+64.7}{2700-0.947 \rho_{d}} \pm 20 \%$ 
$\lambda_{e}=0.7 \log S_{r}+1 \rightarrow$ Fine - grained $\operatorname{soil}\left(S_{r}>0.05\right)$

$\lambda_{e}=\log S_{r}+1 \rightarrow$ Coarse - grained soil $\left(S_{r}>0.1\right)$

where $\lambda_{s}, \lambda_{w}$ and $n$ in Eq. (3a) represent the thermal conductivity of the solid particles, the thermal conductivity of water and the soil porosity, respectively. The terms $\rho_{d}$ in Eq. (3b) and $S_{r}$ in Eqs. (3c) and (3d) are instead the dry density and the degree of saturation of the soil, respectively. Inspection of Eqs. (2) and (3) indicated that the model proposed by Johansen [21] does not provide a unique value of the dry thermal conductivity (i.e., considering the $20 \%$ tolerance) and it requires the determination of the thermal conductivity of the solid particles, which may be a challenging task from the experimental point of view. The model also provides two distinct equations (i.e., Equations (3c) and (3d)) for fine- and coarse-grained soils, thus hindering the direct application of the model to soils with well-graded granularities (i.e., soils composed by comparable percentages of the different granulometric fractions).

Another empirical model for predicting the thermal conductivity of unsaturated geomaterials has been proposed by Liuzzi et al. [22], who measured the moisture dependent thermal conductivity of different types of clay mixes either left unstabilised or stabilised with 5\% of hydrated lime by dry weight. Both unstabilised and stabilised geomaterial samples were equalised at different levels of relative humidity ranging between 35 and $85 \%$ and at a constant temperature of $23{ }^{\circ} \mathrm{C}$. After equalisation, the thermal conductivity was determined by means of the transient plane source device ISOMET 2104. To interpret the experimental data, Liuzzi et al. [22] also proposed a simple empirical thermal conductivity model which relates the ratio $\frac{\lambda}{\lambda_{\text {dry }}}$ between the moisture-dependent thermal conductivity $\lambda$ and the dry thermal conductivity $\lambda_{\text {dry }}$ with the degree of saturation $S_{r}$ by means of the following mathematical expression:

$\frac{\lambda}{\lambda_{\mathrm{dry}}}=1+m_{f} S_{r}$

where $m_{f}$ is a model parameter named the moisture factor. Inspection of Eq. (4) indicates that the model proposed by Liuzzi et al. [22] does not account for the influence of the dry density on the thermal conductivity of the material. The model presented in the present work extends the formulation proposed by Liuzzi et al. [22] to overcome this limitation, as it will be described in the following section.

\section{Thermal Conductivity Model}

The present work proposes a simple relationship between the ratio $\frac{\lambda}{\lambda_{\mathrm{dry}}}$ of the moisture-dependent thermal conductivity $\lambda$ over the dry thermal conductivity $\lambda_{\text {dry }}$, the degree of saturation $S_{r}$ and the dry density $\rho_{d}$, which is defined as follows:

$\frac{\lambda}{\lambda_{\mathrm{dry}}}=1+m_{f} S_{r}+m_{d} \frac{w \rho_{d}}{\rho_{s}}$

where $w$ is the water content, $\rho_{s}$ is the density of the solid particles and $m_{d}$ is a dry density factor (i.e., a fitting parameter). Equation 5 correctly predicts that the moisture-dependent thermal conductivity $\lambda$ becomes equal to the dry thermal conductivity $\lambda_{\text {dry }}$ as both the degree of saturation and water content are equal to zero. Note that the model predicts a constant thermal conductivity under dry conditions and this is regardless of the level of dry density. A more sophisticated approach would have considered the dependency of the dry thermal conductivity $\lambda_{\text {dry }}$ on the dry density. However, this approach would have required further experimental testing and the introduction of additional model parameters, thus compromising the primary feature of the proposed model, which is its simplicity. Despite this limitation, the model is still capable of providing an accurate estimation of the thermal conductivity of various geomaterials, as it will be shown in the following section.

\section{Model Validation}

The proposed thermal conductivity model has been validated against experimental data published by Hall and Allinson [23], Mansour et al. [24], Song et al. [25], Bruno et al. [26] and Tang et al. [5].

Hall and Allinson [23] measured the moisture dependent thermal conductivity on three earth mixes composed by a 14-6.3 mm rounded pea gravel, $5 \mathrm{~mm}$-down medium grade grit sand and a silty clay. In particular, Hall and Allinson [23] named these three mixes as 433, 613 and 703 according to the proportion of each component with the first, second and third number being the mass proportion in units of ten of pea gravel, grit sand and silty clay, respectively. All soil mixes were stabilised with the addition of $6 \%$ by total dry mass of ordinary CEM IIa Portland cement, mixed with the optimum water content of $8 \%$ and then compacted according to the standard Proctor procedure as prescribed by the norm BS 1377-4 [27]. The thermal conductivity was measured by means of a computer-controlled P.A. Hilton B480 heat flow meter apparatus with descending vertical heat flow in agreement with the ISO 8301 [28]. Figure 1a-c show respectively the experimental values of thermal conductivity for the soil mixes 433, 613 and 703 plotted against the corresponding degree of saturation and dry density and together with the model prediction. Inspection of Fig. 1 suggests the excellent capability of the model of reproducing the thermal conductivity of the different soil mixes. This was achieved 


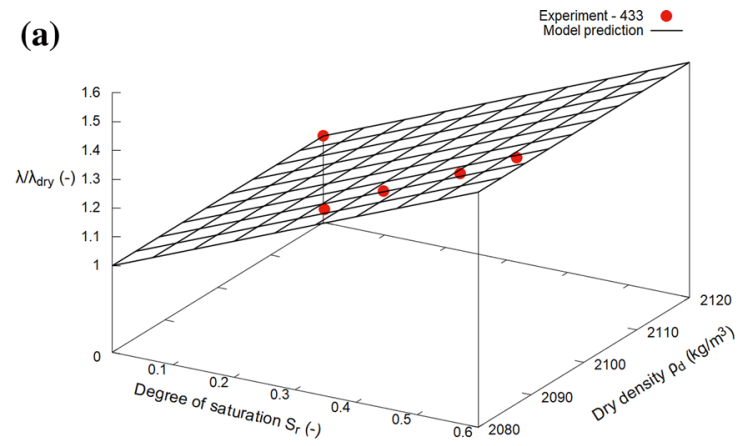

(b)
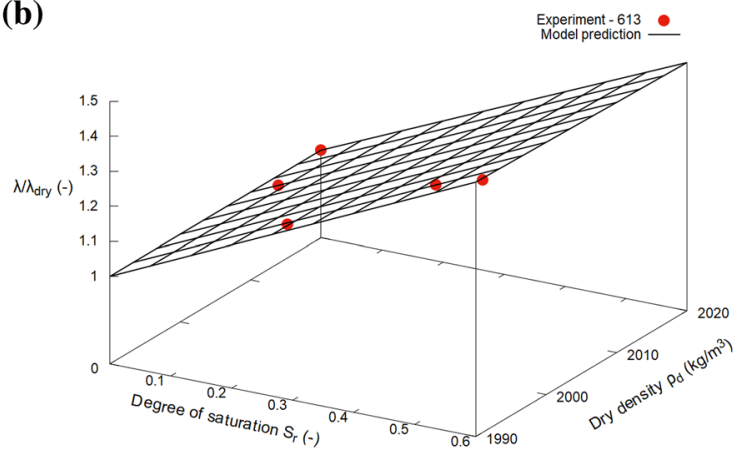

(c)

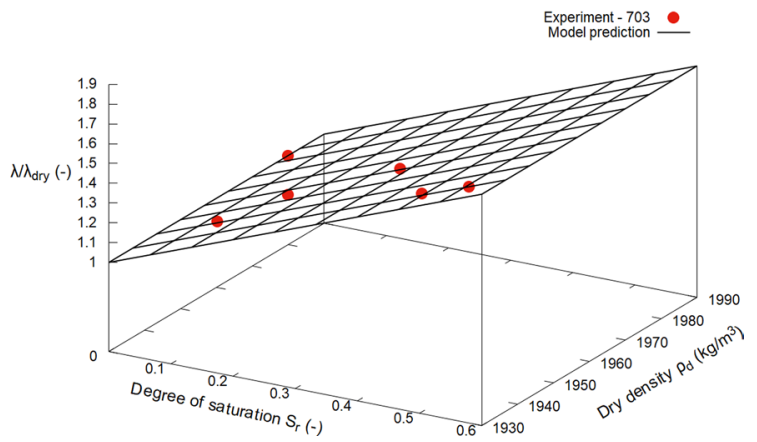

Fig. 1 Model validation against experimental data by Hall and Allinson [23] on earth materials 433 (a), 613 (b) and 733 (c)

regardless of the specific grain size distribution of the tested material and over a range of dry density between $1939 \mathrm{~kg} /$ $\mathrm{m}^{3}$ and $2230 \mathrm{~kg} / \mathrm{m}^{3}$ and a degree of saturation varying from 0 up to 0.6 .

Mansour et al. [24] performed hot wire tests to determine the thermal conductivity of a soil composed of $59.9 \%$ of clay and silt, $39.1 \%$ of sand and $1.0 \%$ of gravel with a liquid limit of $22.4 \%$ and a plastic index of $6.6 \%$. The soil was mixed with a water content of $13 \%$ and then compacted at different dry densities ranging from $1577 \mathrm{~kg} / \mathrm{m}^{3}$ to $2147 \mathrm{~kg} /$ $\mathrm{m}^{3}$. Measurements of thermal conductivity were taken by means of the hot wire method after the soil samples were left to equalise until the desired water content was reached, thus resulting in a variation of the degree of saturation from 0.076 up to 0.256 . Figure 2 shows the experimental results

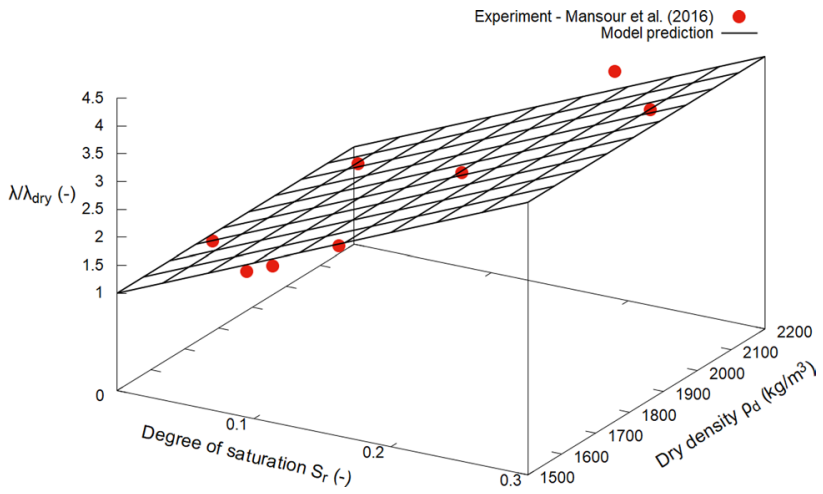

Fig. 2 Model validation against experimental data by Mansour et al. [24] on a silty clay soil

from the thermal conductivity tests performed by Mansour et al. [24] together with the model simulations. Inspection of Fig. 2 confirms the good capability of the model of estimating the thermal conductivity of a relatively fine soil compacted at different dry densities and equalised at various levels of degree of saturation.

Song et al. [25] measured the thermal conductivity of a Xyanyang clay with a liquid limit of $37 \%$, a plasticity index of $13.9 \%$ and a specific gravity of the solid particles $G_{s}$ equal to 2.74. Song et al. [25] used the Hot Disk Transient Plane Source technique to determine the thermal conductivity of soil samples compacted at different dry densities ranging from $1550 \mathrm{~kg} / \mathrm{m}^{3}$ to $2070 \mathrm{~kg} / \mathrm{m}^{3}$ and at various levels of water content ranging from 3 to $24 \%$ (i.e., corresponding to a variation of degree of saturation from 0.107 up to 0.980 ). Figure 3 shows the experimental data by Song et al. [25] plotted together with the corresponding model predictions. Also in this case, the model well reproduces the thermal conductivity of the tested Xyanyang clay samples with a very good fit of the experimental data.

Bruno et al. [26] measured the moisture dependent thermal conductivity of earth samples composed by $0.4 \%$ of

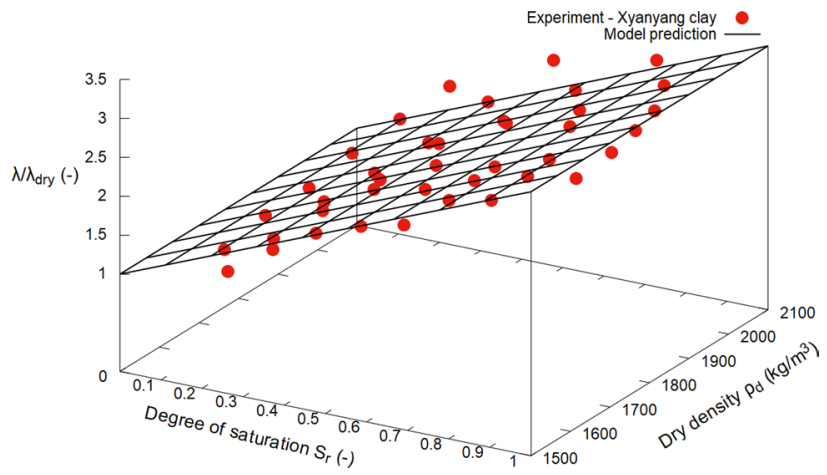

Fig. 3 Model validation against experimental data by Song et al. [25] on a Xyanyang clay 
gravel, $40.4 \%$ of sand, $42.9 \%$ of silt and $16.3 \%$ of illitic clay hypercompacted at a pressure of $100 \mathrm{MPa}$ at the optimum water content of $5.2 \%$. More details on the hypercompaction procedure can be found in Bruno et al. [29] and Bruno et al. [30]. After compaction, three different sets of hypercompacted earth samples were left to equalise inside a climatic chamber at a constant temperature of $25{ }^{\circ} \mathrm{C}$ and at the three levels of the relative humidity of 25,62 and $95 \%$. After equalisation, three measurements of thermal conductivity were taken by means of the Hot Disk Transient Plane Source technique on hypercompacted earth samples for each level of relative humidity. To minimise the effect of different hygrothermal conditions in the laboratory, thermal conductivity measurements were directly taken inside the climatic chamber. Results from this experimental campaign are reported in Fig. 4 together with the model prediction. Note that Bruno et al. [26] did not measure the dry thermal conductivity $\lambda_{\text {dry }}$, which is here extrapolated by assuming a linear relationship between the experimental moisturedependent thermal conductivity and the degree of saturation. Inspection of Fig. 4 shows the good capability of the model of reproducing the thermal conductivity of earth materials compacted at very high dry densities up to $2310 \mathrm{~kg} / \mathrm{m}^{3}$ and equalised to a degree of saturation ranging from 0.346 up to 0.580 . Note that the model validation against the data by Bruno et al. [26] is not as strong as that of the other data sets and this is related to the limited number of thermal conductivity measurements. Nevertheless, it is still worth showing that the proposed model can correctly predict the thermal conductivity of geomaterials at a level of dry density up to $2310 \mathrm{~kg} / \mathrm{m}^{3}$ which is more common in soft rocks rather than compacted soils. This further reiterates the versatility of the model's applications.

Tang et al. [5] measured the thermal conductivity of compacted bentonite, which is a soil that can be used as a buffer material for high-level radioactive waste disposal. In

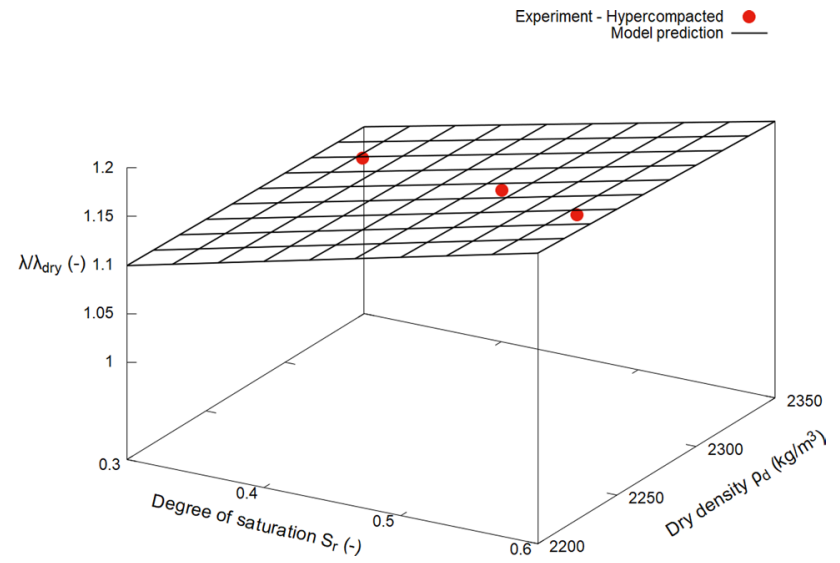

Fig. 4 Model validation against experimental data by Bruno et al. [26] on hypercompacted sandy silt samples particular, Tang et al. [5] tested an MX80 bentonite from Wyoming and purchased from CETCO Europe Ltd. The tested bentonite has a liquid limit of $520 \%$ and a plastic index of $478 \%$, thus attaining a very high plasticity compared with the soils tested by Hall and Allinson [23], Mansour et al. [24], Song et al. [25] and Bruno et al. [26]. Tang et al. [5] used the sensor KD2 from Decagon Devices Inc. to determine the thermal conductivity of the MX80 bentonite compacted at a dry density ranging from $1465 \mathrm{~kg} / \mathrm{m}^{3}$ up to $1801 \mathrm{~kg} / \mathrm{m}^{3}$ and equalised at a degree of saturation ranging from 0.29 up to 0.86 . Results from Tang et al. [5] are plotted in Fig. 5 together with the model predictions. Once again, Fig. 5 shows the good ability of the model of capturing the thermal conductivity of a fine and expansive bentonitic clay with a very high plasticity over the tested ranges of both dry density and degree of saturation.

In conclusion, the good capability of the model in reproducing the thermal conductivity of various unsaturated geomaterials is estimated by means of the Root Mean Square Error RMSE, which is given by the following expression:

$\mathrm{RMSE}=\sqrt{\frac{\sum_{i=1}^{m}\left[\left(\frac{\lambda}{\lambda_{\mathrm{dry}}}\right)_{\bmod , i}-\left(\frac{\lambda}{\lambda_{d r y}}\right)_{\exp , i}\right]^{2}}{m}}$

where $\left(\frac{\lambda}{\lambda_{\mathrm{dry}}}\right)_{\bmod , i}$ and $\left(\frac{\lambda}{\lambda_{\mathrm{dry}}}\right)_{\exp , i}$ are respectively the predicted and experimental ratios between the moisture-dependent and dry thermal conductivities while $m$ is the number of measurements performed for each data set. Values of RMSE for all the data sets considered in the present work together with the numerical values of the model parameters $m_{f}$ and $m_{d}$ are summarised in Table 1. In particular, the model parameters $m_{f}$ and $m_{d}$ were calibrated by means of a least-square regression of the available data. These latter values further confirm that the degree of saturation tend to have a more remarkable influence on the variation of the moisture-dependent thermal

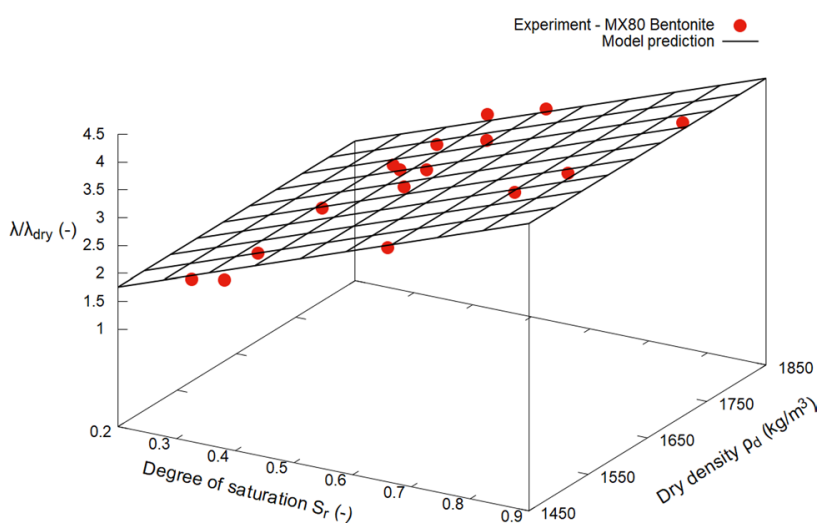

Fig. 5 Model validation against experimental data by Tang et al. [5] on an MX80 Bentonite 
Table 1 Values of $m_{f}, m_{d}$ and RMSE for all data sets

\begin{tabular}{llcll}
\hline References & Soil & $m_{f}$ & $m_{d}$ & RMSE \\
\hline Hall and Allinson [23] & Mix 433 & 0.858 & $2.54 \times 10^{-4}$ & 0.030 \\
& Mix 613 & 0.793 & $3.48 \times 10^{-3}$ & 0.030 \\
& Mix 703 & 1.21 & $1.48 \times 10^{-3}$ & 0.032 \\
Mansour et al. [24] & Silty clay soil & 10.45 & $1.13 \times 10^{-1}$ & 0.337 \\
Song et al. [25] & Xyanyang clay & 2.14 & $1.50 \times 10^{-2}$ & 0.185 \\
Bruno et al. [26] & Hypercompacted sandy silt & 0.324 & $2.51 \times 10^{-2}$ & 0.005 \\
Tang et al. [5] & MX80 Bentonite & 3.75 & $2.54 \times 10^{-1}$ & 0.229 \\
\hline
\end{tabular}

conductivity compared with that of the dry density. However, the model still successfully captured the limited effect of dry density on the thermal conductivity.

Inspection of Table 1 also indicates that the quality of the model predictions is highly accurate for the data published by Hall and Allinson [23] and Bruno et al. [26]. In the former case, the excellent predictive capability of the model can be attributed to the low scatter of the experimental data while in the latter case, the limited number of experimental data has facilitated the calibration of both moisture and dry density factors. Also note that, in both datasets of Hall and Allinson [23] and Bruno et al. [26], the good quality of the prediction is also related to the limited range of dry densities investigated by these two experimental works. The quality of the model predictions progressively reduces for the data published by Song et al. [25], Tang et al. [5] and Mansour et al. [24] but still maintaining a satisfactorily level of accuracy. The larger deviation between the predicted and experimental values of thermal conductivity for these three data sets can be attributed to the more significant dispersion of the data.

\section{Conclusions}

The paper proposed a simple thermal conductivity model for various types of geomaterials in an unsaturated state. The model postulated that the ratio between the moisturedependent thermal conductivity and the dry thermal conductivity depends on the degree of saturation and dry density by means of only two model parameters, namely the moisture factor $m_{f}$ and the dry density factor $m_{d}$. The proposed model has been validated against five different sets of experimental data consisting of measurements of thermal conductivity conducted on earth samples compacted at different dry densities and degree of saturation. The main outcomes of the present study can be summarised as follows:

- The model well predicts the thermal conductivity of earth materials over a broad range of degrees of saturation spanning from dry to almost saturated conditions.
- Model predictions are highly accurate over a wide range of dry densities ranging from $1465 \mathrm{~kg} / \mathrm{m}^{3}$ (typical of geotechnical applications) to $2310 \mathrm{~kg} / \mathrm{m}^{3}$ (representative of earth dwellings or deep geothermal reservoirs).

- The effect of the degree of saturation on the thermal conductivity is more significant than that of the dry density and this is due to the high thermal conductivity of water compared to that of air.

- An increase of dry density (and hence a reduction of the porosity) often induces an increase of the degree of saturation, thus inducing a combined action on the thermal conductivity of the geomaterials, which is well captured by the proposed model.

- The model well predicts the moisture-dependent thermal conductivity of a broad range of soil granularities varying from fine and expansive bentonitic clays to coarse sandy soil mixes.

- The model provided reliable thermal conductivity estimations of data obtained using various laboratory techniques (e.g., heat flow meter apparatus, hot wire method, hot disk transient plane source technique, KD2 thermal properties analyser), thus further confirming the validity of these measuring techniques.

Interestingly, the proposed model can be applied on a very broad range of engineering, geophysical and geoenvironmental applications (e.g., heat exchange with geothermal reservoirs, safe disposal of radioactive waste in bentonitic buffers, thermal performance of earth materials used for building constructions, optimised management of cultivations and crops, etc.). Finally, the simplicity of the model facilitates its implementation in Finite Element codes for the numerical study of heat transfer occurring in geomaterials in a partially saturated state.

\section{Funding None.}

\section{Compliance with Ethical Standards}

Conflict of Interest The authors declare that they have no conflict of interest. 
Open Access This article is licensed under a Creative Commons Attribution 4.0 International License, which permits use, sharing, adaptation, distribution and reproduction in any medium or format, as long as you give appropriate credit to the original author(s) and the source, provide a link to the Creative Commons licence, and indicate if changes were made. The images or other third party material in this article are included in the article's Creative Commons licence, unless indicated otherwise in a credit line to the material. If material is not included in the article's Creative Commons licence and your intended use is not permitted by statutory regulation or exceeds the permitted use, you will need to obtain permission directly from the copyright holder. To view a copy of this licence, visit http://creativecommons.org/licenses/by/4.0/.

\section{References}

1. Eugster WJ, Sanner B (2007) Technological status of shallow geothermal energy in Europe. In Proceedings European geothermal congress (Vol. 8)

2. Bertermann D, Klug H, Morper-Busch L (2015) A pan-European planning basis for estimating the very shallow geothermal energy potentials. Renew Energy 75:335-347

3. Sáez Blázquez C, Farfán Martín A, Martín Nieto I, GonzalezAguilera D (2017) Measuring of thermal conductivities of soils and rocks to be used in the calculation of a geothermal installation. Energies 10(6):795

4. Vieira A, Maranha J, Christodoulides P, Alberdi-Pagola M, Loveridge F, Nguyen F et al (2017) Characterisation of ground thermal and thermo-mechanical behaviour for shallow geothermal energy applications. Energies 10(12):2044

5. Tang AM, Cui YJ, Le TT (2008) A study on the thermal conductivity of compacted bentonites. Appl Clay Sci 41(3-4):181-189

6. Lee JO, Choi H, Lee JY (2016) Thermal conductivity of compacted bentonite as a buffer material for a high-level radioactive waste repository. Ann Nucl Energy 94:848-855

7. Yoon S, Cho W, Lee C, Kim GY (2018) Thermal conductivity of Korean compacted bentonite buffer materials for a nuclear waste repository. Energies 11(9):2269

8. Zhang L, Gustavsen A, Jelle BP, Yang L, Gao T, Wang Y (2017) Thermal conductivity of cement stabilized earth blocks. Constr Build Mater 151:504-511

9. Alsabry A, Backiel-Brzozowska B, Nikitsin VI (2020) Dependencies for determining the thermal conductivity of moist capillaryporous materials. Energies 13(12):3211

10. Zhao SX, Ta N, Wang XD (2017) Effect of temperature on the structural and physicochemical properties of biochar with apple tree branches as feedstock material. Energies 10(9):1293

11. Yang X, Wang H, Strong PJ, Xu S, Liu S, Lu K et al (2017) Thermal properties of biochars derived from waste biomass generated by agricultural and forestry sectors. Energies 10(4):469

12. Zhao D, Qian X, Gu X, Jajja SA, Yang R (2016) Measurement techniques for thermal conductivity and interfacial thermal conductance of bulk and thin film materials. J Electron Packaging 138(4)

13. Bouguerra A, Aït-Mokhtar A, Amiri O, Diop MB (2001) Measurement of thermal conductivity, thermal diffusivity and heat capacity of highly porous building materials using transient plane source technique. Int Commun Heat Mass Transfer 28(8):1065-1078

14. Franco A (2007) An apparatus for the routine measurement of thermal conductivity of materials for building application based on a transient hot-wire method. Appl Therm Eng 27(14-15):2495-2504

15. Dong Y, McCartney JS, Lu N (2015) Critical review of thermal conductivity models for unsaturated soils. Geotech Geol Eng 33(2):207-221

16. Wang J, He H, Dyck M, Lv J (2020) A review and evaluation of predictive models for thermal conductivity of sands at full water content range. Energies 13(5):1083

17. De Vries DA (1963) Thermal properties of soils. Physics of plant environment

18. Mickley AS (1951) The thermal conductivity of moist soil. Trans Am Inst Electr Eng 70(2):1789-1797

19. Woodside WMJH, Messmer JH (1961) Thermal conductivity of porous media I Unconsolidated sands. J Appl Phys 32(9): 1688-1699

20. McGaw R (1969) Heat conduction in saturated granular materials. Highway Research Board Special Report (103)

21. Johansen O (1977) Thermal conductivity of soils. PhD thesis, University of Trondheim - Cold Regions Research and Engineering Lab Hanover NH, Trondheim, Norway

22. Liuzzi S, Hall MR, Stefanizzi P, Casey SP (2013) Hygrothermal behaviour and relative humidity buffering of unfired and hydrated lime-stabilised clay composites in a Mediterranean climate. Build Environ 61:82-92

23. Hall M, Allinson D (2009) Assessing the effects of soil grading on the moisture content-dependent thermal conductivity of stabilised rammed earth materials. Appl Therm Eng 29(4):740-747

24. Mansour MB, Jelidi A, Cherif AS, Jabrallah SB (2016) Optimizing thermal and mechanical performance of compressed earth blocks (CEB). Constr Build Mater 104:44-51

25. Song X, Fan H, Liu J, Yang X (2020) An improved thermal conductivity model for unsaturated clay. KSCE J Civil Eng 1-8

26. Bruno AW, Gallipoli D, Perlot C, Kallel H (2020) Thermal performance of fired and unfired earth bricks walls. J Build Eng 28:101017

27. Standard B (1990) BS 1377-4-1990, Methods of test for Soils for civil engineering purposes-Part4: Compaction-related tests. London, British Standard institute

28. ISO 8301 (1991) Thermal insulation-Determination of steadystate thermal resistance and related properties-Heat flow meter apparatus

29. Bruno AW, Gallipoli D, Perlot C, Mendes J (2017) Mechanical behaviour of hypercompacted earth for building construction. Mater Struct 50(2):160

30. Bruno AW, Perlot C, Mendes J, Gallipoli D (2018) A microstructural insight into the hygro-mechanical behaviour of a stabilised hypercompacted earth. Mater Struct 51(1):32

Publisher's Note Springer Nature remains neutral with regard to jurisdictional claims in published maps and institutional affiliations. 